\title{
Renal cancer: overdiagnosis and overtreatment
}

\author{
Giuseppe Rosiello $^{1,2} \cdot$ Alessandro Larcher $^{1,2} \cdot$ Francesco Montorsi $^{1,2} \cdot$ Umberto Capitanio $^{1,2}$
}

Published online: 12 August 2021

(C) The Author(s), under exclusive licence to Springer-Verlag GmbH Germany, part of Springer Nature 2021

Renal cell carcinoma (RCC) accounts for approximately $4.1 \%$ of all new cancers, with a median age at diagnosis of 64 years. In 2020, around 73,750 new cases were expected to be diagnosed in the United States, with an age-adjusted rate of 16.3 cases $/ 100.000$ persons [1]. Worldwide, the incidence of RCC has been rising [2] due to the widespread use of cross-sectional imaging performed for other reasons (e.g. hypertension, diabetes, etc.) [3]. As a consequence, a significant increase in incidental detection of renal masses has been observed over time, especially in elderly patients, who are more likely to undergo radiological investigations for other health-related problems [4]. It is of note that early detection of kidney tumors has been accompanied by a clinical-stage migration toward earlier tumor stages. Specifically, despite locally advanced RCC continues to be diagnosed in a high proportion of patients, with up to $17 \%$ harbouring metastatic disease at the time of diagnosis [5], the rates of $\mathrm{cT}_{1}$ renal masses increased from $40 \%$ in 1992 to roughly $70 \%$ in 2015 [6] Nevertheless, several reports demonstrated a lack of declining mortality rates for localized RCC over time [7], and the cancer-specific mortality rate of patients harbouring $\mathrm{T}_{1 \mathrm{a}}$ disease remains below $5 \%$ at 5 years. [4] Such a controversial relationship between increasing detection rates of small renal masses (SRMs), but relatively stable survival outcomes highlights the importance of carefully evaluating and discussing the phenomenon of early detection in RCC, and make the concept of "overdiagnosis" of great importance in this context.

"Overdiagnosis" can be defined as the detection of a renal mass that would not cause any symptoms or clinical progression, if never diagnosed [8]. Therefore, the overdiagnosis of

Umberto Capitanio

capitanio.umberto@hsr.it

1 Department of Urology, IRCCS San Raffaele Scientific Institute, Via Olgettina 60, 20132 MI Milan, Lombardia, Italy

2 Division of Experimental Oncology, URI, Urological Research Institute, IRCCS San Raffaele Scientific Institute, Milan, Italy a small, silent renal mass crashes against our limited ability in determining the nature and the aggressiveness of the incidentally detected mass. Indeed, despite some improvements have been done in the field of nuclear imaging and radiomics [9], the current technology is limited and still, more than $20 \%$ of surgery are done for SRMs, which result totally benign at final pathology.

During the last two decades, the incidence of RCC increased from 9.2 to 12.5 cases per 100.000 persons/year. Notably, over $45 \%$ of newly diagnosed RCC have tumor size $\leq 4 \mathrm{~cm}$, and this percentage is on the rise. Based on what was previously stated, it is not surprising that the incidence of $\mathrm{T}_{1 \mathrm{a}} \mathrm{RCC}$ recorded the highest increase over time: $+50 \%$ since the beginning of the new millennium. [10] However, more in-depth analyses of age-specific data are necessary to better understand this phenomenon and its implications in clinical practice. Specifically, despite the incidence rates of SRMs also increased in young adults (from 0.1 to 0.4 cases per 100.000 persons/year), [11] such estimates are much lower than what was recorded in the general population. [2] Conversely, data regarding elderly patients are extremely different and deserve particular attention. Indeed, approximately $75 \%$ of newly diagnosed RCC are reported in patients over the age of 60 . The age-standardised incidence rate in this patient population is as high as 35.0 cases per 100.000 persons/year, and the highest rate is recorded in patients aged $\geq 75$ years. [2] Moreover, the incidence of SRMs is 30 -fold higher in $\geq 75$ years old than in younger patients. [12] These data demonstrate the magnitude of the problem, and should not be underestimated but, instead, they should sensitize the urological community about the important implications that they may have, in a global and comprehensive evaluation of the phenomenon. Indeed, the potential consequences of overdiagnosis may be significant and include psychological and behavioural effects of disease labelling, physical harms and side effects of unnecessary tests or treatments, increased financial costs to individuals and wasted resources and opportunity costs to the health system. Last but not least, overdiagnosis may lead to an increased risk of overtreatment. 
In RCC, overdiagnosis does not imply invasive detection manoeuvres, as happens for instance in other urological settings (e.g. prostatic biopsy). Therefore, more importantly than overdiagnosis, the most detrimental and dangerous aspect in RCC is the decision after the diagnosis or in other words-the potential "overtreating". "Overtreatment" is a partially overlapped phenomenon which implies the treatment (and the related consequences) of cancer which wouldn't have caused any symptom or cancer-related death. Overtreatment comes immediately after our inaccuracy in determining the characteristics of the mass. Even in the case of malignant pathology, the natural history of small low-grade kidney cancer is indolent-in most of the cases-if well balanced with life expectancy, comorbidities and competing risks mortality. Indeed, the majority of overdiagnoses happen in old and comorbid patients, where the balance between pro and cons is completely different relative to the diagnosis of RCC in a middle-aged and healthy patient. While postoperative complications are the most obvious danger resulting from surgical "overtreatment" of patients with SRMs, the decrease in renal function is also well-established [13, 14], and the risk resulting from kidney surgery needs to be evaluated, especially in those at higher risk of long-term renal impairment, such as elderly or comorbid patients. Moreover, studies exploring the benefits of nephrectomy in patients older than 75 years with clinically localized $\mathrm{cT}_{1}$ renal masses demonstrated no superiority of definitive treatment (either partial or radical approach) compared to active surveillance in terms of cancer-specific survival, due to decreased life expectancy in this patient population. In other words, most of these patients would not live long enough to benefit from surgery [4].

In the current issue of World Journal of Urology, review papers and original articles provide recent data which may limit the effect of overdiagnosis and overtreatment in the kidney cancer setting. Specifically, the natural history of untreated kidney cancer [15], the importance of frailty and comorbidities for clinical decision making [16, 17], the role of contrast-enhanced ultrasound [18], the novelties in radiomics and biomarkers and the current ongoing active surveillance protocols were summarized and critically analyzed [19-21].

Funding This research did not receive any specific grant from funding agencies in the public, commercial, or not-for-profit sectors.

\section{Declarations}

Conflict of interest None to declare.

\section{References}

1. Motzer RJ, Jonasch E, Agarwal N, Bhayani S, Bro WP, Chang SS et al (2017) Kidney cancer, version 2.2017: clinical practice guidelines in oncology. J Natl Compr Cancer Netw 15:804-834. https://doi.org/10.6004/jnccn.2017.0100

2. Capitanio U, Bensalah K, Bex A, Boorjian SA, Bray F, Coleman $\mathrm{J}$ et al (2019) Epidemiology of renal cell carcinoma. Eur Urol 75:74-84. https://doi.org/10.1016/j.eururo.2018.08.036

3. Chow W-H, Dong LM, Devesa SS (2010) Epidemiology and risk factors for kidney cancer. Nat Rev Urol 7:245-257. https://doi. org/10.1038/nrurol.2010.46

4. Sun M, Becker A, Tian Z, Roghmann F, Abdollah F, Larouche A et al (2014) Management of localized kidney cancer: calculating cancer-specific mortality and competing risks of death for surgery and nonsurgical management. Eur Urol 65:235-241. https://doi. org/10.1016/j.eururo.2013.03.034

5. Capitanio U, Montorsi F (2016) Renal cancer. Lancet 387:894906. https://doi.org/10.1016/S0140-6736(15)00046-X

6. Kane CJ, Mallin K, Ritchey J, Cooperberg MR, Carroll PR (2008) Renal cell cancer stage migration: analysis of the national cancer data base. Cancer 113:78-83. https://doi.org/10.1002/cncr.23518

7. Sun M, Thuret R, Abdollah F, Lughezzani G, Schmitges J, Tian Z et al (2011) Age-adjusted incidence, mortality, and survival rates of stage-specific renal cell carcinoma in North America: a trend analysis. Eur Urol 59:135-141. https://doi.org/10.1016/j.eururo. 2010.10.029

8. Welch HG, Black WC (2010) Overdiagnosis in cancer. J Natl Cancer Inst 102:605-613. https://doi.org/10.1093/jnci/djq099

9. Leong JY, Wessner CE, Kramer MR, Forsberg F, Halpern EJ, Lyshchik A et al (2020) Superb microvascular imaging improves detection of vascularity in indeterminate renal masses. J Ultrasound Med. https://doi.org/10.1002/jum.15299

10. Palumbo C, Pecoraro A, Knipper S, Rosiello G, Luzzago S, Deuker $\mathrm{M}$ et al (2020) Contemporary age-adjusted incidence and mortality rates of renal cell carcinoma: analysis according to gender, race, stage, grade, and histology. Eur Urol Focus. https://doi.org/ 10.1016/j.euf.2020.05.003

11. Palumbo C, Pecoraro A, Rosiello G, Luzzago S, Deuker M, Stolzenbach F et al (2020) Renal cell carcinoma incidence rates and trends in young adults aged 20-39 years. Cancer Epidemiol 67:101762. https://doi.org/10.1016/j.canep.2020.101762

12. King SC, Pollack LA, Li J, King JB, Master VA (2014) Continued increase in incidence of renal cell carcinoma, especially in young patients and high grade disease: United States 2001 to 2010. J Urol 191:1665-1670. https://doi.org/10.1016/j.juro.2013.12.046

13. Bhindi B, Lohse CM, Schulte PJ, Mason RJ, Cheville JC, Boorjian SA et al (2019) Predicting renal function outcomes after partial and radical nephrectomy (figure presented). Eur Urol 75:766-772. https://doi.org/10.1016/j.eururo.2018.11.021

14. Rosiello G, Capitanio U, Larcher A (2019) Acute kidney injury after partial nephrectomy: transient or permanent kidney damage? Impact on long-term renal function. Ann Transl Med 7:S317S317. https://doi.org/10.21037/atm.2019.09.156

15. McAlpine K, Finelli A (2021) Natural history of untreated kidney cancer. World J Urol. https://doi.org/10.1007/s00345-020-03578-1

16. Walach MT, Wunderle MF, Haertel N et al (2021) Frailty predicts outcome of partial nephrectomy and guides treatment decision towards active surveillance and tumor ablation. World J Urol. https://doi.org/10.1007/s00345-020-03556-7

17. Courcier J, De La Taille A, Lassau N et al (2021) Comorbidity and frailty assessment in renal cell carcinoma patients. World $\mathrm{J}$ Urol. https://doi.org/10.1007/s00345-021-03632-6

18. Bertelli E, Palombella A, Sessa F et al (2021) Contrastenhanced ultrasound (CEUS) imaging for active surveillance 
of small renal masses. World J Urol. https://doi.org/10.1007/ s00345-021-03589-6

19. Patel SH, Singla N, Pierorazio PM (2021) Decision-making in active surveillance in kidney cancer: current trends and future urine and tissue markers. World J Urol. https://doi.org/10.1007/ s00345-021-03786-3

20. Rebez G, Pavan N, Mir MC (2021) Available active surveillance follow-up protocols for small renal mass: a systematic review. World J Urol. https://doi.org/10.1007/s00345-020-03581-6
21. Kuusk T, Neves JB, Tran M et al (2021) Radiomics to better characterize small renal masses. World J Urol. https://doi.org/10.1007/ s00345-021-03602-y

Publisher's Note Springer Nature remains neutral with regard to jurisdictional claims in published maps and institutional affiliations. 